\title{
Avaliação da atividade antioxidante em diferentes extratos da polpa e sementes da romã (Punica granatum, L.)
}

\author{
Fernanda Archilla Jardini", Jorge Mancini Filho \\ Departamento de Alimentos e Nutrição Experimental, Faculdade de Ciências Farmacêuticas, \\ Universidade de São Paulo
}

F.A. Jardini

Departamento de Alimentos e Nutrição

Experimental

Faculdade de Ciências Farmacêuticas

Universidade de São Paulo

Av. Prof. Lineu Prestes, 580, BI. 14 05508-900 - São Paulo - SP, Brasil

E-mail: fjardini@usp.br
Avaliou-se a atividade antioxidante dos extratos etéreo, alcoólico e aquoso obtidos da polpa e das sementes da romã (Punica granatum, L.), utilizando-se dois sistemas: 1) co-oxidação do $\beta$-caroteno/ácido linoléico e 2) Rancimat ${ }^{\circledR}$. Inicialmente foram determinados os compostos redutores presentes na polpa $(1.214 \mathrm{mg} / 100 \mathrm{~g})$ e nas sementes (1.732 mg/100 g) e, em seqüência, avaliou-se nos extratos, através de cromatografia em camada delgada (CCD), a presença de compostos fenólicos com atividade antioxidante. Os extratos aquosos tanto da polpa quanto das sementes apresentaram as maiores porcentagens de inibição da oxidação: 87,31\% e 93,08\%, respectivamente. Através da avaliação cinética pelo teste de cooxidação com $\beta$-caroteno e ácido linoléico, pôde-se verificar que a alta porcentagem da atividade antioxidante dos extratos aquosos é devida à capacidade de inibir a oxidação nas fases inicial e mais avançada do processo oxidativo, agindo por mecanismos primário e secundário. Todos os extratos (etéreo, alcoólico e aquoso) apresentaram elevada capacidade em prolongar o periodo de indução da oxidação, medida pelo aparelho Rancimat ${ }^{\circledR}$, sendo, ainda, significativamente $(p<0,05)$ maiores que os resultados obtidos com o antioxidante sintético butil-hidroxianisol (BHA).

\section{INTRODUÇÃO}

A oxidação é uma reação que pode ocorrer nos alimentos provocando a perda do valor nutritivo pela decomposição dos ácidos graxos e a formação de compostos que podem reagir com outros componentes dos alimentos e também serem prejudiciais para os organismos humano e animal (Marinova, Yanishilieva, 2003; Mathew, Abraham, 2005).
Para evitar o desenvolvimento da reação oxidativa, os antioxidantes são empregados como aditivos alimentares. Os antioxidantes sintéticos butil-hidróxi-tolueno (BHT), o butilhidróxi-anisol (BHA) e o terc-butil-hidroquinona (TBHQ) são amplamente utilizados pela indústria alimentícia. Porém, estudos em animais evidenciaram que a exposição aguda e prolongada destes compostos levou ao desenvolvimento de tumores de fígado, pâncreas e glândulas (Hirose et al., 1981), aumento da formação de $\mathrm{H}_{2} \mathrm{O}_{2}$ nos microssomos, alterando 
as funções hepáticas (Rossing, Kahl, Hildebrandt, 1985), carcinogênese no estômago de ratos (Ito et al., 1983) e adenomas e carcinomas em células hepáticas (Würtzen, Olsen, 1986). Frente a estas evidências, desenvolveu-se a pesquisa sobre os antioxidantes de origem natural, com a perspectiva de sua utilização como aditivos alimentares (Nissen et al., 2001; Bernal Gómez, Mendonça Júnior, Mancini- Filho, 2003; Sallam, Ishioroshi, Samejima, 2004; Reedy, Urooj, Kumar, 2005), como também pelas evidências de que estes compostos podem atuar em benefício da saúde (Bub et al., 2003).

A romã (Punica granatum, L.) é uma fruta originária da região do Oriente Médio. A árvore cresce em regiões áridas e a produção do fruto se dá no período de setembro a fevereiro (Martins, 1995). O suco da romã apresenta em sua composição compostos fenólicos como: antocianinas (delfinidina, cianidina e pelargonidina), quercetina, ácidos fenólicos (caféico, catequínico, clorogênico, orto e paracumárico, elágico, gálico e quínico) e taninos (punicalagina) (Artik, Murakami, Mori, 1998; Noda et al., 2002; Poyrazoglu, Gökmen, Artik, 2002; Seeram et al., 2005). Trabalhos experimentais demonstraram que os compostos fenólicos da romã apresentaram influência sobre fatores biológicos, como a atenuação de fatores aterogênicos (Aviran et al., 2000; Aviran, Dornfeld, 2001), modulação das respostas antiinflamatórias (Ross et al., 2001) e de enzimas do sistema de defesa antioxidante endógeno (superóxido dismutase, catalase e glutationa peroxidase) (Ajaikumar $e t$ al., 2005). Também os flavonóides extraídos do suco fermentado e do óleo da romã tiveram atividade inibitória das enzimas oxidantes ciclooxigenase e lipooxigenase (Schubert, Lanski, Neeman, 1999).

Segundo os dados da CEAGESP da cidade de São Paulo, foram comercializados nos anos de 2001 a 2004 somente na cidade de São Paulo, em média 200 toneladas da fruta. Frente à presença de compostos fenólicos na fruta e à importância que este fato apresenta tanto para a aplicação em alimentos quanto para a saúde, nos propusemos a verificar o potencial antioxidante da polpa e sementes da romã, em extratos obtidos a partir de solventes de diferentes polaridades empregando-se o sistema modelo de co-oxidação com $\beta$-caroteno e ácido linoléico e o aparelho Rancimat ${ }^{\mathbb{Q}}$.

\section{MATERIAL E MÉTODOS}

\section{Material}

\section{Reagentes e solventes}

Os solventes de grau analítico utilizados foram os ácidos bórico e sulfúrico, os álcoois etílico e metílico, clorofórmio e éter etílico da marca $S y n t h^{\circledR}$. Os reagentes ácido linoléico ( $99 \%$ de pureza), $\beta$-caroteno ( $95 \%$ de pureza), os antioxidantes sintéticos butil-hidróxi- tolueno (BHT) e butil-hidróxi- anisol (BHA), a catequina ( $98 \%$ de pureza), a sílica-gel $60 \mathrm{G}$ e o Tween ${ }^{\circledR} 40$ da marca Sigma ${ }^{\circledR}$. O reagente Folin-Ciocalteau utilizado foi da Merck $^{\circledR}$. O óleo de soja puro, sem adição de antioxidantes foi cedido pela empresa Cargil Agrícola S/A.

\section{Amostra}

As romãs, provenientes da cidade de Petrolina (PE), foram adquiridas na Companhia de Entrepostos e Armazéns Gerais do Estado de São Paulo (CEAGESP) da cidade de São Paulo, no mês de janeiro. As frutas foram lavadas e abertas à temperatura ambiente, retirando-se a parte interna que foi colocada sobre uma peneira plástica de $17 \mathrm{~cm}$ de diâmetro, posicionada sobre um bequer de $500 \mathrm{~mL}$ envolto em papel alumínio, protegendo da luz. O material foi friccionado contra a peneira, sendo dividido em polpa (coletada na forma de suco) e sementes, que foram moídas com auxílio de um moinho analítico e padronizadas em tamis de mesh 32. A polpa e as sementes moídas foram submetidas à liofilização, sendo após estocadas em congelador à temperatura de $-18^{\circ} \mathrm{C}$.

\section{MÉTODOS}

\section{Composição centesimal}

Os conteúdos de umidade, lípides e resíduo mineral foram determinados seguindo-se a metodologia descrita pelo Instituto Adolfo Lutz (1985). O conteúdo de proteínas foi determinado segundo o método descrito na AOAC (1995). O conteúdo de carboidratos totais foi realizado por exclusão do valor obtido pela somatória dos valores de umidade, lípides, resíduo mineral e proteína (Instituto Adolfo Lutz, 1985).

\section{Conteúdo de fenólicos totais}

A determinação dos conteúdos de compostos fenólicos totais da polpa e sementes da romã foi realizada de acordo com o método espectrofotométrico descrito por Singleton, Orthofer e Lamuela-Raventós (1999), utilizando o reagente de Folin-Ciocalteau como agente redutor, e a catequina (concentração de $1 \mathrm{mg} / \mathrm{mL}$ ) para construção da curva- padrão. As amostras e a catequina foram diluídas em solução hidroalcoólica a $80 \%$. Os resultados foram expressos em $\mathrm{mg}$ de compostos/100 $\mathrm{g}$ de amostra.

\section{Obtenção dos extratos}

A obtenção dos extratos alcoólico e aquoso da polpa, e etéreo, alcoólico e aquoso das sementes da romã 
foi realizada pelo método de extração seqüencial. As extrações foram realizadas na proporção de 1:20 (amostra: solvente) e os solventes utilizados foram o éter etílico, o álcool etílico e a água destilada, seguindo-se esta ordem de polaridade para a extração. As amostras foram homogeneizadas durante uma hora, sendo, em seguida, filtradas em funil de Büchner com auxílio de uma bomba de vácuo. O resíduo proveniente da filtração foi seco, pesado e submetido à extração com o solvente subseqüente. Os extratos obtidos foram coletados em frasco de vidro âmbar e estocados em congelador a $-18{ }^{\circ} \mathrm{C}$ (Torres et al.,2002).

\section{Separação dos compostos fenólicos por cromatografia em camada delgada (CCD)}

Os extratos obtidos da polpa e das sementes da romã foram submetidos à análise em cromatografia em camada delgada (CCD), para prévia identificação de compostos fenólicos com capacidade antioxidante. O método seguido foi o descrito por Duve e White (1991), utilizando-se placas de sílica-gel ( $0,25 \mathrm{~mm}$ de espessura), às quais foram aplicados os extratos no volume de $200 \mu \mathrm{L}$ (concentrações relativas descritas na Tabela I). A revelação foi feita empregando-se dois agentes reveladores: (SR 1) constituído de mistura de soluções aquosas de cloreto férrico e ferricianeto de potássio ( $1 \%$ cada uma) e (SR 2) constituído da mistura das soluções de $\beta$-caroteno $(0,3 \mathrm{mg} / \mathrm{mL}$ diluídos em clorofórmio $)$ e ácido linoléico $(0,7 \mathrm{mg} / \mathrm{mL}$ diluído em etanol). As bandas reveladas foram medidas e os resultados expressos pelo fator de retenção (Rf).

\section{Determinação da atividade antioxidante}

\section{Ensaio em sistema $\beta$-caroteno e ácido linoléico}

A avaliação da atividade antioxidante foi realizada segundo o método espectrofotométrico $(470 \mathrm{~nm})$ originalmente descrito por Marco (1968) e modificado por Miller
(1971). Foi preparada uma emulsão, com $20 \mu \mathrm{L}$ de solução de $\beta$-caroteno a $20 \%, 40 \mu \mathrm{L}$ de ácido linoléico, $200 \mathrm{mg}$ de Tween $40^{\circledR}$ e $120 \mathrm{~mL}$ de água destilada mantida sob atmosfera de oxigênio (30 minutos) e a absorbância inicial foi ajustada entre 0,6 e 0,7. Em tubos de ensaio, foram adicionados $5 \mathrm{~mL}$ da emulsão preparada e o antioxidante padrão BHT e os extratos nos volumes de 50, 100 e $200 \mu \mathrm{L}$, nas concentrações descritas na Tabela I. O sinergismo entre as amostras e o BHT também foi avaliado. Os tubos foram mantidos em banho a $50^{\circ} \mathrm{C}$, realizando-se leituras a cada 15 minutos em um total de duas horas. Os resultados foram expressos em \% de inibição da oxidação.

Estudo cinético da atividade antioxidante do ensaio em sistema $\beta$-caroteno e ácido linoléico

A estimativa da eficiência da atividade antioxidante dos extratos da polpa e sementes da romã foi avaliada pelo método do cálculo das tangentes das curvas obtidas no sistema $\beta$-caroteno e ácido linoléico, segundo descrito em Giada (2006) e obtendo-se os fatores cinéticos F1 e F2. A medida dos fatores cinéticos foi baseada em dois momentos distintos da reação que ocorre no método de Marco (1968) e Miller (1971): F1 foi calculado no tempo compreendido entre 15 a 45 minutos, pois é a parte da reação em que a formação de radicais peróxidos é maior, avaliando-se a capacidade do antioxidante em agir por mecanismos primários; F2 foi calculado no tempo compreendido entre 75 a 105 minutos, pois nesta etapa avançada da reação é possível avaliar a eficiência do antioxidante em agir por mecanismos secundários, interferindo nas reações de formação de produtos secundários da reação de oxidação. Foi considerado o valor referente às tangentes o coeficiente resultante da divisão entre o cateto oposto (obtido pela diferença entre as absorbâncias dentro do tempo avaliado) e cateto adjacente (obtido pela diferença entre os tempos de 15 a 45 minutos (F1) e 75 a 105 minutos (F2)), como mostrado na Figura 1.

TABELA I - Concentrações $(\mu \mathrm{g})$ do antioxidante sintético BHT e dos extratos etéreo, alcoólico e aquoso da polpa e sementes da romã,nos respectivos volumes avaliados nos ensaios de cromatografia em camada delgada e no ensaio em sistema $\beta$-caroteno e ácido linoléico

\begin{tabular}{lcccc}
\hline & $25 \mu \mathrm{L}$ & $50 \mu \mathrm{L}$ & $100 \mu \mathrm{L}$ & $200 \mu \mathrm{L}$ \\
\hline Alcoólico - polpa & 0,77 & 1,5 & 3,09 & 6,18 \\
Aquoso - polpa & 10,8 & 21,61 & 43,22 & 86,44 \\
Etéreo - semente & 0,25 & 0,51 & 1,02 & 2,04 \\
Alcoólico - semente & 1,81 & 3,61 & 7,23 & 14,46 \\
Aquoso - semente & 8,79 & 17,57 & 35,15 & 70,3 \\
BHT & 25 & 50 & 100 & 200 \\
\hline
\end{tabular}




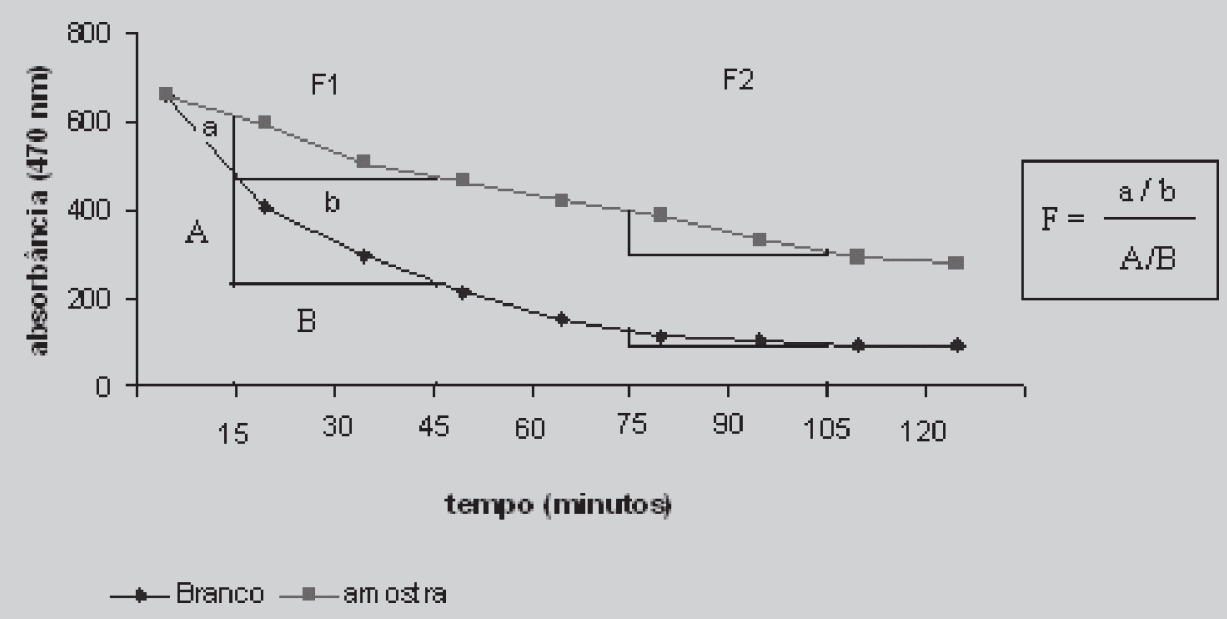

FIGURA 1- Modelo de curva de caimento obtida no sistema modelo $\beta$-caroteno e ácido linoléico, para obtenção das tangentes do cálculo dos fatores cinéticos F1 e F2, em que (a) e (A) são os catetos opostos, obtidos através da diferença das leituras das absorbâncias (da amostra e do branco, respectivamente); (b) e (B) são os catetos adjacentes, obtidos através das diferença entre os tempos (respectivamente, da amostra e do branco), de acordo com o fator cinético calculado.

\section{Ensaio em aparelho Rancimat ${ }^{\circledR}$}

A atividade antioxidante dos extratos da polpa e sementes da romã foi avaliada em meio lipídico, utilizando-se o aparelho Rancimat $^{\circledR}$ (modelo 743 - Metrhom $^{\circledR}$ (Herissau - SW)). O fluxo de oxigênio foi programado para $20 \mathrm{~L} / \mathrm{h}$ e a temperatura de $110{ }^{\circ} \mathrm{C}$. O substrato lipídico utilizado foi o óleo de soja, isento de antioxidantes (empresa Cargill Agrícola S/A) e o antioxidante sintético BHA escolhido como padrão comparativo. As concentrações testadas foram de 100 e $200 \mathrm{ppm}$ e os resultados expressos em porcentagem de atividade antioxidante, calculada a partir do período de indução (P.I.) obtidos em horas.

\section{Avaliação estatística}

Todas as análises foram realizadas em triplicata, sendo os resultados expressos em média \pm desvio-padrão. O programa utilizado para a execução das análises foi o SigmaStat, versão 1.0, realizando-se a análise de variância pelo teste ANOVA e a comparação entre as médias dos resultados pelo método Student-Newman-Keuls, fixandose o nível de significância de $\mathrm{p}<0,05$.

\section{RESULTADOS E DISCUSSÃO}

A composição centesimal da polpa e sementes da romã está apresentada na Tabela II.

Pôde-se observar que a polpa apresentou alto teor
TABELA II - Composição centesimal da polpa e sementes da romã, expressos em base úmida

\begin{tabular}{lcc}
\hline & POLPA (\%) & SEMENTE (\%) \\
\hline Umidade & $64,09 \pm 1,16$ & $38,30 \pm 0,44$ \\
Lípides & $0,24 \pm 0,00$ & $14,06 \pm 0,16$ \\
Proteína & $1,128 \pm 0,02$ & $2,81 \pm 0,08$ \\
Resíduo mineral & $2,09 \pm 0,07$ & $0,86 \pm 0,01$ \\
Carboidratos & 32,3 & 43,97 \\
\hline
\end{tabular}

$\mathrm{n}=3$; resultados expressos em média \pm desvio-padrão.

de umidade $(64,09 \%)$ e que os valores de carboidratos estimados foram altos, tanto para a polpa $(32,3 \%)$ quanto para as sementes $(43,97 \%)$. Também, verificou-se que as sementes apresentaram valor considerável de lípides $(14,06 \%)$.

Com o objetivo de estimar a presença de compostos com capacidade redutora, foi realizado o ensaio com o reagente de Folin Ciocalteau. Os resultados do ensaio, para a polpa e sementes da romã, são apresentados na Tabela III.

De acordo com os resultados, as sementes apresentaram conteúdo maior de compostos redutores (1732 $\mathrm{mg}$ de compostos redutores/100 $\mathrm{g}$ de amostra) em relação à polpa (1214 mg de compostos redutores/100 g de amostra). Os resultados encontram-se abaixo dos descritos por Gil et al. (2000), em que foram comparados os conteúdos de compostos redutores do suco industrializado (2.566 mg de compostos redutores/L) e o obtido ma- 
TABELA III - Resultados da análise de fenólicos totais da polpa e sementes da romã expressos em $\mathrm{mg}$ de compostos redutores $/ 100 \mathrm{~g}$ de amostra

\begin{tabular}{lc}
\hline & mg de compostos redutores $/ 100 \mathrm{~g}$ de amostra \\
\hline POLPA & $1.214 \pm 102,66$ \\
SEMENTE & $1.732 \pm 12,66$ \\
\hline
\end{tabular}

$\mathrm{n}=3$, resultados expressos em média \pm desvio- padrão. Nível de significância de $5 \%(\mathrm{p}<0,05)$.

nualmente (2.117 mg de compostos redutores/L). O maior valor de compostos redutores no suco obtido industrialmente foi atribuído ao processo de extração, em que a casca e o olbedo (partes adstringentes e ricas em taninos, compostos fenólicos altamente hidroxilados), não são removidos para o processamento. Ainda, fatores como grau de maturação, região de cultivo e diferença entre espécies da fruta e a presença de algumas vitaminas (especialmente a vitamina $C$ ) e alguns açúcares redutores podem participar do valor total, quando é utilizado o reagente de Folin-Ciocalteau (Singleton, Orthofer, Lamuela-Raventós, 1999; Moure et al., 2001).

$\mathrm{Na}$ Tabela IV estão apresentados os Rf (fator de retenção) dos extratos da polpa e das sementes da romã obtidos por cromatografia em camada delgada. Nesta tabela podem-se identificar as correspondências entre os Rf dos compostos fenólicos caracterizados pelo sistema revelador SR 1 com os Rf's dos compostos antioxidantes caracterizados pelo sistema revelador SR 2.

De acordo com os resultados apresentados na Tabela IV, observou-se que todos os extratos apresentaram pelo menos uma banda pelo SR1, identificando a presença de compostos fenólicos em todos os extratos. Também foi verificado que todas as bandas foram igualmente reveladas pelo SR2, indicando capacidade antioxidante. O extrato aquoso das sementes foi o extrato com maior número de bandas reveladas $(\mathrm{Rf}=0,83 ; 0,26$ e 0,22$)$.

Frente às evidências de presença de compostos com capacidade antioxidante, foram realizados testes com os extratos, para a avaliação da atividade antioxidante, através do ensaio empregando-se $\beta$-caroteno e ácido linoléico. Os resultados da atividade antioxidante estão expressos nas Tabelas V (polpa) e VI (sementes).

Pôde-se observar, de acordo com os resultados expressos nas Tabelas V e VI, que os extratos aquosos foram os que apresentaram os maiores valores de porcentagem de inibição da oxidação. Em estudos de atividade antioxidante realizados com extratos de diferentes polaridades, utilizando-se espécies de algas (Vidal et al., 2001; Linares et al., 2004), os resultados obtidos com os extratos aquosos foram
TABELA IV - Valores de Rf (fator de retenção) obtidos para os extratos da polpa e sementes da romã, através de cromatografia em camada delgada (CCD)

\begin{tabular}{lcc}
\hline & \multicolumn{2}{c}{$\mathrm{Rf}$} \\
\cline { 2 - 3 } EXTRATO & $\mathrm{SR} 1$ & $\mathrm{SR} 2$ \\
\hline Polpa & & \\
Alcoólico & 0,32 & 0,32 \\
& 0,21 & 0,21 \\
Aquoso & 0,44 & 0,44 \\
Sementes & 0,32 & 0,32 \\
Etéreo & 0,82 & 0,82 \\
& & \\
Alcoólico & 0,39 & 0,39 \\
& 0,25 & 0,25 \\
Aquoso & 0,83 & 0,83 \\
& 0,26 & 0,26 \\
& 0,22 & 0,22 \\
\hline
\end{tabular}

SR1 = sistema revelador 1 (solução aquosa $1 \%$ de $\mathrm{FeCl}_{3}$ e $\left.\mathrm{K}_{3}(\mathrm{CN})_{6}\right)$; SR2 = sistema revelador 2 (solução $\beta$-caroteno e ácido linoléico)

também bastante significativos.

O solvente extrator constitui fator relevante e deve ser levado em conta, em função da quantidade e quais tipos de compostos presentes na amostra são desejados extrair para se avaliar a atividade antioxidante (Naczk, Shahidi, 2004; Zhou, Yu, 2004). Lapornik, Prosek e Wondra (2005) verificaram que a maior atividade antioxidante era condizente com o maior conteúdo de antocianinas presentes nos três extratos (metílico, etílico e aquoso) avaliados, de amora e casca de uva. Efeito semelhante do conteúdo de compostos extraídos influenciando positivamente na atividade antioxidante foi relatado por Lo e Cheung (2005).

$\mathrm{O}$ extrato aquoso das sementes apresentou diferença significativa da porcentagem de inibição da oxidação, na concentração máxima testada $(70,4 \mu \mathrm{g}=93,08 \%)$ em relação ao mesmo extrato, obtido da polpa $(86,4 \mu \mathrm{g}=87,31 \%)$.

Todos os extratos apresentaram atividade sinergística, notada mais significativamente nas menores concentrações testadas. Para as concentrações máximas os valores de inibição da oxidação foram altos e tanto o BHT quanto os extratos avaliados individualmente já apresentaram saturação do sistema antioxidante, não sendo possível, portanto, identificar acréscimo na inibição da oxidação. Isto fica claro no extrato aquoso da semente, como pode ser visto na Tabela VI. 
TABELA V - Porcentagem de inibição da oxidação para o padrão BHT e para os extratos da polpa da romã e para a associação entre os extratos e BHT, no sistema $\beta$-caroteno e ácido linoléico

\begin{tabular}{|c|c|c|c|c|c|c|}
\hline \multicolumn{7}{|c|}{ POLPA } \\
\hline$\mu \mathrm{L}$ & & & & & & \\
\hline Extrato & 50 & $25+25$ & 100 & $50+50$ & 200 & $100+100$ \\
\hline Alcoólico & $47,09^{a} \pm 1,25$ & $71,73^{b} \pm 1,25$ & $65,59^{a} \pm 4,11$ & $83,51^{b} \pm 2,94$ & $76,39^{a} \pm 1,25$ & $87,97^{b} \pm 0,82$ \\
\hline Aquoso & $74,61^{a} \pm 0,94$ & $82,35^{b} \pm 1,41$ & $81,98^{\mathrm{a}} \pm 1,41$ & $86,93^{b} \pm 1,25$ & $87,31^{\mathrm{a}} \pm 0,47$ & $91,39^{b} \pm 2,62$ \\
\hline BHT & $73,80^{\mathrm{a}} \pm 2,72$ & & $82,56^{\mathrm{a}} \pm 1,45$ & & $87,64^{a} \pm 0,77$ & \\
\hline
\end{tabular}

$\mathrm{n}=3$; resultados expressos em média \pm desvio- padrão. $\mathrm{a}=$ diferença significativa $(\mathrm{p}<0,05 \%)$ para a mesma amostra, com aumento do volume; $\mathrm{b}=$ sinergismo entre a amostra e o BHT $(\mathrm{p}<0,05 \%)$. Concentrações $(\mu \mathrm{g})$ relativas aos volumes testados: BHT $(25 \mu \mathrm{L}$ $=25 \mu \mathrm{g} ; 50 \mu \mathrm{L}=50 \mu \mathrm{g} ; 100 \mu \mathrm{L}=100 \mu \mathrm{g} ; 200 \mu \mathrm{L}=200 \mu \mathrm{g})$; extrato alcoólico $(25 \mu \mathrm{L}=0,75 \mu \mathrm{g} ; 50 \mu \mathrm{L}=1,5 \mu \mathrm{g} ; 100 \mu \mathrm{L}=3,0$ $\mu \mathrm{g} ; 200 \mu \mathrm{L}=6,0 \mu \mathrm{g})$; extrato aquoso $(25 \mu \mathrm{L}=10,8 \mu \mathrm{g} ; 50 \mu \mathrm{L}=21,6 \mu \mathrm{g} ; 100 \mu \mathrm{L}=43,2 \mu \mathrm{g} ; 200 \mu \mathrm{L}=86,4 \mu \mathrm{g})$.

TABELA VI - Porcentagem de inibição da oxidação para o padrão BHT e para os extratos das sementes da romã, e para a associação entre os extratos e BHT, no sistema $\beta$-caroteno e ácido linoléico

\section{SEMENTE}

\begin{tabular}{lcccccc}
\hline \multicolumn{1}{c}{$\mu \mathrm{L}$} & & & & & \\
Extrato & 50 & $25+25$ & 100 & $50+50$ & 200 & $100+100$ \\
\hline Etéreo & $66,79^{\mathrm{a}} \pm 1,89$ & $74,13^{\mathrm{b}} \pm 7,12$ & $77,80^{\mathrm{a}} \pm 2,49$ & $77,80^{\mathrm{b}} \pm 2,49$ & $83,67^{\mathrm{a}} \pm 0,47$ & $79,17 \pm 2,36$ \\
Alcoólico & $34,19^{\mathrm{a}} \pm 5,72$ & $64,91 \pm 2,87$ & $61,40^{\mathrm{a}} \pm 1,25$ & $73,27 \pm 0,94$ & $71,35^{\mathrm{a}} \pm 0,94$ & $82,00^{\mathrm{b}} \pm 5,73$ \\
Aquoso & $75,89^{\mathrm{a}} \pm 1,25$ & $85,15^{\mathrm{b}} \pm 0,47$ & $89,12^{\mathrm{a}} \pm 1,70$ & $90,32^{\mathrm{b}} \pm 3,40$ & $93,08 \pm 1,25$ & $93,87^{\mathrm{b}} \pm 2,16$ \\
BHT & $72,78^{\mathrm{a}} \pm 3,48$ & & $80,68^{\mathrm{a}} \pm 1,43$ & & $86,52^{\mathrm{a}} \pm 1,12$ & \\
\hline
\end{tabular}

$\mathrm{n}=3$; resultados expressos em média \pm desvio- padrão. $\mathrm{a}=$ diferença significativa $(\mathrm{p}<0,05 \%)$ para a mesma amostra, com aumento do volume; $\mathrm{b}=$ sinergismo entre a amostra e o BHT $(\mathrm{p}<0,05 \%)$. Concentrações $(\mu \mathrm{g})$ relativas aos volumes testados: BHT $(25 \mu \mathrm{L}=25 \mu \mathrm{g} ; 50 \mu \mathrm{L}=50 \mu \mathrm{g} ; 100 \mu \mathrm{L}=100 \mu \mathrm{g} ; 200 \mu \mathrm{L}=200 \mu \mathrm{g})$; extrato etéreo $(25 \mu \mathrm{L}=0,25 \mu \mathrm{g} ; 50 \mu \mathrm{L}$ $=0,5 \mu \mathrm{g} ; 100 \mu \mathrm{L}=1,0 \mu \mathrm{g} ; 200 \mu \mathrm{L}=2,0 \mu \mathrm{g}) ;$ extrato alcoólico $(25 \mu \mathrm{L}=1,8 \mu \mathrm{g} ; 50 \mu \mathrm{L}=3,6 \mu \mathrm{g} ; 100 \mu \mathrm{L}=7,2 \mu \mathrm{g} ; 200 \mu \mathrm{L}$ $=14,4 \mu \mathrm{g})$; extrato aquoso $(25 \mu \mathrm{L}=8,8 \mu \mathrm{g} ; 50 \mu \mathrm{L}=17,6 \mu \mathrm{g} ; 100 \mu \mathrm{L}=35,2 \mu \mathrm{g} ; 200 \mu \mathrm{L}=70,4 \mu \mathrm{g})$.

TABELA VII - Parâmetros cinéticos do potencial antioxidante no sistema $\beta$-caroteno e ácido linoléico, para o BHT, os extratos da polpa e para a associação entre extratos e BHT

F1 F2

\begin{tabular}{lcccccccccccc}
\hline \multicolumn{1}{c}{$\mu \mathrm{L}$} & & & & & & & & & & & \\
Extrato & 50 & $25+25$ & 100 & $50+50$ & 200 & $100+100$ & 50 & $25+25$ & 100 & $50+50$ & 200 & $100+100$ \\
\hline Alcoólico & 0,4 & 0,2 & 0,23 & 0,14 & 0,18 & 0,09 & 2,15 & 1,22 & 1,21 & 0,85 & 0,8 & 0,53 \\
Aquoso & 0,09 & 0,09 & 0,06 & 0,06 & 0,03 & 0,02 & 0,7 & 0,59 & 0,45 & 0,45 & 0,3 & 0,25 \\
BHT & 0,18 & & 0,12 & & 0,07 & & 0,89 & & 0,57 & & 0,43 & \\
\hline
\end{tabular}

$\mathrm{F} 1$ = fator cinético 1 (tempo compreendido entre 15 a 45 minutos) e $\mathrm{F} 2$ = fator cinético 2 (tempo compreendido entre 75 a 105 minutos). O nível de significância adotado foi de $5 \%(\mathrm{p}<0,05)$. Concentrações $(\mu \mathrm{g})$ relativas aos volumes testados: BHT $(25 \mu \mathrm{L}=25 \mu \mathrm{g} ; 50 \mu \mathrm{L}=50 \mu \mathrm{g} ; 100 \mu \mathrm{L}=100 \mu \mathrm{g} ; 200 \mu \mathrm{L}=200 \mu \mathrm{g})$; extrato alcoólico $(25 \mu \mathrm{L}=0,75 \mu \mathrm{g} ; 50 \mu \mathrm{L}=1,5 \mu \mathrm{g} ; 100 \mu \mathrm{L}$ $=3,0 \mu \mathrm{g} ; 200 \mu \mathrm{L}=6,0 \mu \mathrm{g})$; extrato aquoso $(25 \mu \mathrm{L}=10,8 \mu \mathrm{g} ; 50 \mu \mathrm{L}=21,6 \mu \mathrm{g} ; 100 \mu \mathrm{L}=43,2 \mu \mathrm{g} ; 200 \mu \mathrm{L}=86,4 \mu \mathrm{g})$.

A avaliação da eficiência do antioxidante relacionada ao seu possível mecanismo de ação foi mostrada no estudo de cinética da curva de decaimento da atividade antioxidante. Os resultados estão expressos nas Tabelas VII (polpa) e VIII (sementes).

A eficiência do antioxidante é tanto maior quanto mais distante os valores de F1 e F2 se encontrarem do valor 1,0 (Moreira, Mancini-Filho, 2003). Pelos resultados obtidos pôde-se verificar que todos valores de F1 atribuídos ao BHT e aos extratos encontraram-se abaixo de 1,0. Já para os valores de F2, alguns extratos apresentaram valores acima de 1,0, decaindo com o aumento da concentra- 
TABELA VIII - Parâmetros cinéticos do potencial antioxidante no sistema $\beta$-caroteno e ácido linoléico, para o BHT, os extratos das sementes e para a associação entre extratos e BHT

F1

F2

\begin{tabular}{lcccccccccccc}
\hline \multicolumn{1}{c}{$\mu \mathrm{L}$} & & & & & & & & & & & \\
Extrato & 50 & $25+25$ & 100 & $50+50$ & 200 & $100+100$ & 50 & $25+25$ & 100 & $50+50$ & 200 & $100+100$ \\
\hline téreo & 0,41 & 0,24 & 0,16 & 0,09 & 0,12 & 0,04 & 1,26 & 1,02 & 0,78 & 0,66 & 0,57 & 0,32 \\
Alcoólico & 0,38 & 0,19 & 0,26 & 0,13 & 0,17 & 0,1 & 1,63 & 0,82 & 1,16 & 0,41 & 0,67 & 0,36 \\
Aquoso & 0,16 & 0,11 & 0,11 & 0,06 & 0,07 & 0,05 & 0,66 & 0,45 & 0,39 & 0,31 & 1,37 & 0,19 \\
BHT & 0,18 & & 0,12 & & 0,07 & & 0,89 & & 0,57 & & 0,43 & \\
\hline
\end{tabular}

$\mathrm{F} 1$ = fator cinético 1 (tempo compreendido entre 15 a 45 minutos) e F2 = fator cinético 2 (tempo compreendido entre 75 a 105 minutos). O nível de significância adotado foi de $5 \%(\mathrm{p}<0,05)$. Concentrações $(\mu \mathrm{g})$ relativas aos volumes testados: BHT $(25 \mu \mathrm{L}=25 \mu \mathrm{g} ; 50 \mu \mathrm{L}=50 \mu \mathrm{g} ; 100 \mu \mathrm{L}=100 \mu \mathrm{g} ; 200 \mu \mathrm{L}=200 \mu \mathrm{g})$; extrato etéreo $(25 \mu \mathrm{L}=0,25 \mu \mathrm{g} ; 50 \mu \mathrm{L}=0,5 \mu \mathrm{g}$; $100 \mu \mathrm{L}=1,0 \mu \mathrm{g} ; 200 \mu \mathrm{L}=2,0 \mu \mathrm{g})$; extrato alcoólico $(25 \mu \mathrm{L}=1,8 \mu \mathrm{g} ; 50 \mu \mathrm{L}=3,6 \mu \mathrm{g} ; 100 \mu \mathrm{L}=7,2 \mu \mathrm{g} ; 200 \mu \mathrm{L}=14,4 \mu \mathrm{g})$; extrato aquoso $(25 \mu \mathrm{L}=8,8 \mu \mathrm{g} ; 50 \mu \mathrm{L}=17,6 \mu \mathrm{g} ; 100 \mu \mathrm{L}=35,2 \mu \mathrm{g} ; 200 \mu \mathrm{L}=70,4 \mu \mathrm{g})$.

ção testada. Nos extratos aquosos da polpa (Tabela VII) e das sementes (Tabela VIII), tanto os valores de F1 quanto de F2 foram os menores obtidos. Quando comparados os extratos aquosos com o antioxidante sintético BHT, verifica-se que houve diferença significativa na eficiência dos extratos aquosos da polpa e sementes sobre o BHT, para os valores de F1 e F2. O sinergismo entre as amostras e o antioxidante sintético BHT também foi notado.

Os resultados mostram que os extratos aquosos podem inibir a oxidação tanto por mecanismos primários (caracterizados pela medida de F1) quanto secundários (medidos por F2), fato refletido na alta porcentagem de inibição mostrada nas Tabelas V e VI.

As Tabelas IX e $\mathrm{X}$ trazem as porcentagens de atividade antioxidante baseadas no prolongamento do período de indução, em horas, para os extratos e para o antioxidante sintético BHA no teste sob condições aceleradas de oxidação, utilizando-se o aparelho Rancimat ${ }^{\mathbb{}}$.

$\mathrm{O}$ antioxidante sintético escolhido para a avaliação da atividade antioxidante em meio lipídico foi o BHA, em função deste permitir maior proteção em sistemas oleosos, nos quais a presença da água é baixa, em relação ao antioxidante sintético BHT nas mesmas condições (Guerra, Lajolo, 2005).

De acordo com as Tabelas IX e X, pôde-se verificar que houve diferença significativa entre os extratos e o antioxidante sintético BHA, sendo a diferença tanto maior quanto maior a polaridade do solvente extrator. Assim, o extrato aquoso sobressaiu-se aos demais, apresentando os valores de 47,76\% (100 ppm) e 53,45\% (200 ppm) para o extrato aquoso da polpa e $42,16 \%$ (100 ppm) e 44,31\% (200 ppm) para o extrato aquoso das sementes.

Em meio oleoso, a baixa atividade de água favorece a reação de oxidação, em função da concentração do substrato. Assim, os extratos aquosos apresentam bom desempenho no meio lipídico, pois além da ação dos compostos antioxidantes que formam uma barreira na interface óleo/ ar, impedindo a ação do oxigênio sobre o óleo, a água presente como veículo dos antioxidantes dis-

TABELA IX - Porcentagem de inibição da oxidação referente ao período de indução (em horas) para o antioxidante sintético BHA e os extratos da polpa da romã, pelo método Rancimat ${ }^{\circledR}$

\% de inibição da oxidação

ppm

Extrato

100

200

\begin{tabular}{lcc}
\hline Alcoólico & $41,39^{\mathrm{b}} \pm 12,10$ & $45,82^{\mathrm{b}} \pm 10,27$ \\
Aquoso & $47,76^{\mathrm{b}} \pm 10,83$ & $53,45^{\mathrm{a}, \mathrm{b}} \pm 8,47$ \\
BHA & $11,65 \pm 4,06$ & $14,47 \pm 3,57$
\end{tabular}

$\mathrm{n}=3$; resultados expressos em média \pm desvio- padrão. O nível de significância adotado foi de $5 \%(\mathrm{p}<0,05)$. $\mathrm{a}=$ diferença significativa em relação ao mesmo extrato, na concentração de 100 ppm e b = diferença significativa, em relação ao controle antioxidante sintético BHA, na respectiva concentração. 
TABELA X - Porcentagem de inibição da oxidação referente ao período de indução (em horas), para o antioxidante sintético BHA e os extratos das sementes da romã, pelo método Rancimat ${ }^{\mathbb{R}}$

\% de inibição da oxidação

ppm

Extrato

100

200

\begin{tabular}{lcc}
\hline Etéreo & $9,62 \pm 4,27$ & $19,55^{\mathrm{a}} \pm 14,10$ \\
Alcoólico & $30,47^{\mathrm{b}} \pm 4,61$ & $35,41^{\mathrm{b}} \pm 6,53$ \\
Aquoso & $42,16^{\mathrm{b}} \pm 2,29$ & $44,31^{\mathrm{b}} \pm 3,10$ \\
BHA & $11,65 \pm 4,06$ & $14,47 \pm 3,57$
\end{tabular}

$\mathrm{n}=3$; resultados expressos em média \pm desvio- padrão. O nível de significância adotado foi de $(\mathrm{p}<0,05) .{ }^{\mathrm{a}}=$ diferença significativa em relação ao mesmo extrato, na concentração de 100 ppm e ${ }^{b}=$ diferença significativa, em relação ao controle antioxidante sintético BHA, na respectiva concentração.

persaria o substrato oxidável, através da elevação da atividade de água do óleo (Frankel et al., 1994; Guerra, Lajolo, 2005).

É importante a realização de diferentes testes para a avaliação da atividade antioxidante, para a obtenção de resposta mais precisa sobre a interação dos compostos presentes na amostra com os diferentes radicais gerados durante a reação (Robards, 2003). No teste de oxidação do $\beta$-caroteno e ácido linoléico, pode-se medir na primeira etapa (15 a 45 minutos) a capacidade dos compostos em doar elétrons ou átomos de hidrogênio, prolongando o período de indução e, na segunda etapa (75 a 105 minutos) a interação com os compostos gerados na degradação do ácido linoléico. Já no teste utilizando o aparelho Rancimat ${ }^{\circledR}$, os compostos formados são os voláteis, decorrentes da oxidação numa fase mais adiantada do processo. Entretanto, apesar da variedade de métodos que possibilitam mensurar a atividade antioxidante, os resultados obtidos neste trabalho, ambos os testes indicam a elevada atividade antioxidante dos diferentes extratos obtidos da polpa e sementes da romã. Os diferentes resultados são difíceis de serem comparados (Giada, Mancini- Filho, 2004).

\section{CONCLUSÕES}

A polpa e as sementes da romã apresentam potencial antioxidante, verificado pela presença de compostos com capacidade redutora, identificados pela cromatografia em camada delgada como compostos fenólicos.

Os extratos aquosos da polpa e sementes foram os mais eficazes na atividade antioxidante. $\mathrm{O}$ extrato aquoso das sementes apresentou atividade inibitória da oxidação significativamente maior que a alcançada pelo antioxidante sintético BHT, avaliados pelo ensaio do $\beta$-caroteno e ácido linoléico. Na avaliação cinética, pode-se verificar que os extratos aquosos apresentaram-se eficientes tanto nos períodos iniciais quanto mais adiantados do processo oxidativo. No ensaio em meio lipídico (método Rancimat ${ }^{\mathbb{R}}$ ), o extrato aquoso da polpa apresentou os maiores valores de inibição da oxidação, significativamente maiores em relação ao antioxidante sintético BHA.

\section{AGRADECIMENTOS}

Ao Conselho Nacional de Desenvolvimento Científico e Tecnológico (CNPq), pelo suporte financeiro e concessão da bolsa à pesquisa, à Fundação de Amparo à Pesquisa do Estado de São Paulo (FAPESP), pelo suporte financeiro, e à empresa Cargil Agrícola S.A., pela amostra de óleo de soja isento de antioxidantes.

\section{ABSTRACT
Antioxidant activity evaluation of different polarities extracts by pulp and seeds of pomegranate (Punica granatum, L.)

In etheric, alcoholic and aqueous extracts obtained from pomegranate (Punica granatum, L.) pulp and seeds their antioxidant activity was evaluated by using two systems: co-oxidation of $\beta$-carotene/linoleic acid and 2) Rancimat ${ }^{\circledR}$. First of all, the presence of reducing compounds was detected in the pulp $(1,214 \mathrm{mg} / 100 \mathrm{~g})$ and in the seeds $(1,732 \mathrm{mg} / 100 \mathrm{~g})$. The phenolic compounds with antioxidant activity were then evaluated in the extracts using thin-layer chromatography (TLC). The aqueous extracts from the pulp and the seeds showed the highest antioxidant activity of $87.31 \%$ and of $93.08 \%$, respectively. The kinetic curves obtained by cooxidation of $\beta$-carotene and linoleic acid substrates also showed the highest values in the aqueous. These results were 
correlated with the initial and advanced oxidation process phases under activity by primary and secondary mechanisms. All the extracts showed high antioxidant capacity when submitted to the Rancimat ${ }^{\circledR}$ test. This activity was significantly higher than that presented by the standard synthetic antioxidant butilated hidroxy anysole (BHA).

UNITERMS: Pomegranate. Natural antioxidant. Oxidation. Rancimat ${ }^{\circledR}$.

\section{REFERÊNCIAS BIBLIOGRÁFICAS}

AJAIKUMAR, K.B.; ASHEEF, M.; BABU, B.H.; PADIKKALA, J. The inhibition of gastric mucosal injury by Punica granatum, L. (pomegranate) methanolic extract. J. Ethnopharmacol., Orlando, v. 96, n.1-2, p. 171-176, 2005.

ARTIK, N.; MURAKAMI, H.; MORI, T. Determination of phenolic compounds in pomegranate juice by using HPLC. Fruit Process., Oberhonnefeld- Gierend, v. 12, p. 492499, 1998.

ASSOCIATION OF OFFICIAL ANALYTICAL CHEMISTS. Official methods of analysis of the Association of Official Analytical Chemists. 16.ed. Washington: AOAC, 1995. v.1, p.7. n. 960.52/12.1.07.

AVIRAN, M.; DORNFELD, L.; ROSENBLAT, M.; VOLKOVA, N.; KAPLAN, M.; COLEMAN, R.; HAYEK, T.; PRESSER, D.; FUHRMAN, B. Pomegranate juice consumption reduces oxidative stress, atherogenic modifications to LDL, and platelet aggregation: studies in humans and in atherosclerotic apolipoprotein E- deficient mice. Am. J. Clin. Nutr., Rockville Pike, v. 71, n.5, p. 1062- 1076, 2000.

AVIRAN, M.; DORNFELD, L. Pomegranate juice consumption inhibits serum angiotensin converting enzyme activity abd reduces systolic blood pressure. Atherosclerosis, Oxford, v. 158, n.1, p. 195- 198, 2001.

BERNAL GÓMEZ, M.E.; MENDONÇA- JÚNIOR, C.X.; MANCINI FILHO, J. Estabilidad oxidativa de huevos enriquecidos com ácidos grasos poliinsaturados Omega 3 , frente a antioxidantes naturales. Rev. Bras. Cienc. Farmac., São Paulo, v.39, p. 426- 432, 2003.
BUB, A.; WATZL, B.; BLOCKHAUS, M.; BRIVIBA, K.; LIEGIBEL, U.; MÜLLER, H.; POOL- ZOBEL, B.L.; RECHKEMMER, G. Fruit juice consumption modulates antioxidative status, immune status and DNA damage. $J$. Nutr. Biochem., Stoneham, v. 14, n.2, p. 90-98, 2003.

DUVE, K.J.; WHITE, P.J. Extraction and identification of antioxidants in oats. J. Am. Oil Chem. Soc., Champaign, v. 68, p. $365-370,1991$.

FRANKEL, E.N.; HUANG, S.W.; KANNER, J.; GERMAN, J.B. Interfacial phenomena in the evaluation of antioxidants: bulk oils vs. emulsions. J. Agric. Food Chem., Washington, v.42, n.5, p. 1054- 1059, 1994.

GIADA, M.L.R.; MANCINI- FILHO, J. Avaliação da atividade antioxidante in vitro de compostos fenólicos de alimentos. Nutrire, São Paulo, V.28, , p. 91-107, 2004.

GIADA, M.L.R. Avaliação da capacidade antioxidante dos copostos fenólicos do cotilédone da semente de girssol (Helianthus annuus, L.) rajada. São Paulo, 2006, 206 p. [Tese de doutorado. Faculdade de Ciências Farmacêuticas. Universidade de São Paulo].

GIL, M.I.; TOMÁS- BARBERÁN, F.A.; HESS- PIERCE, B.; HOLCROFT, D.M.; KADER, A.A. Antioxidant activity of pomegranate juice and its relationship with phenolic composition and processing. J. Agric. Food Chem., Washington, v.48, n.10, p. 4581-4589, 2000.

GUERRA, N.B.; LAJOLO, F.M. Ação antioxidante de especiarias face diferentes atividades de água. Cien. Tecnol. Aliment., Campinas, v.25, n.1, p. 45-50, 2005.

HIROSE, M.; HAGIWARA, A.; HASUI,; INOVE, K.; ITO, N. Combinated effects of BHA and other antioxidants in induction of forestomach lesions in rats. Cancer Lett., Shannon, v.30, n.2, p. 169-174, 1986.

INSTITUTO ADOLFO LUTZ. Normas analíticas do Instituto Adolfo Lutz: métodos químicos e físicos para análise de alimentos. 3.ed. São Paulo, 1985. 533 p.

ITO, N.; FUKUSHIMA, S.; HAGIWARA, A.; SHIBATA, M.; OGISO, T. Carcinogenicity of butylated hydroxyanisole in F 344 rats. J. Natl. Cancer Inst., Cary, v.70, p. 343-352, 1983. 
LAPORNIK, B.; PROSEK, M.; WONDRA, A.G. Comparision of extracts prepared from plant by-products using different solvents and extraction time J. Food Eng., London, v.71, n.2, p. 214-222, 2005.

LINARES, A.J.; LOIKKANEM, J.; MANCINI- FILHO, J.; SORIA, R.B.; NOVOA, A.V. Antioxidant and neuroprotective activity of extract from the seaweed Halimeda incrassata (Ellis) Lamouroux, against in vitro and in vivo toxicity induced by methyl- mercury. Vet. Human Tox., Manhattan, v. 46, p. 1-5, 2004.

LO, K.M.; CHEUNG, P.C.K. Antioxidant activity of extracts from the fruiting bodies os Agrocybe aegerita var. alba. Food Chem., Washington, v. 89, n.4, p. 533- 539, 2005.

MARCO, G.J.A rapid method for evaluation of antioxidants. J. Am. Oil Chem. Soc., Champaign, v. 45, p. 594- 598, 1968.

MARINOVA, E.M.; YANISHILIEVA, N.V. Antioxidant activity and mechanisms of action of some phenolic acids at ambient and high temperatures. Food Chem., Washington, v. 81, n.2, p. 189-197, 2003.

MARTINS, E. Plantas medicinais. Viçosa: UFV, 1995. p. 162-163.

MATHEW, S.; ABRAHAM, E.. Studies on the antioxidant activities of cinnamon (Cinnamomum verum) bark extracts, through various in vitro models. Food Chem. Disponível em: $<\mathrm{httm}: / /$ www.sciencedirect.com $>$. Acesso em: 16 de fevereiro de 2005.

MILLER, H.E.A simplified method for the evaluation of antioxidants. J. Am. Oil Chem. Soc., Champaign, v. 48, p.91, 1971.

MOREIRA, A.V.B.; MANCINI- FILHO, J. Atividade antioxidante das especiarias mostarda, canela e erva-doce em sistemas aquoso e lipídico. Nutrire, São Paulo, V.25, p.31-46, 2003.

NACZK, M.; SHAHIDI, F. Extractions and analysis of phenolics in food. J. Chromatogr. A, , v.1054, n.1-2, p.95111,2004.

NISSEN, L.R.; MANSSON, L.; BERTELSEN, G.; HUYHBA., T.; SKIBSTED, L.H. Protection of dehydrated chicken meat by natural antioxidants as evaluated by electron spin resonance spectrometry. J. Agric. Food Chem., Washington, v.48, n.11, p. 5548- 5556, 2001.
NODA, Y.; KANEYUKI, T.; MORI, A.; PACKER, L. Antioxidant activities of pomegranate fruit extract and its anthocyanidins: delphinidin, cyaniding and pelargonidin. $J$. Agric. Food Chem., Washington, v.50, n.1, p. 166-171, 2002.

POYRAZOGLU, E.; GÖKMEN, V.; ARTIK, N. Organic acids and phenolic compounds in pomegranates (Punica granatum, L.) grown in Turkey. J. Food Comp. Anal., Kidlington, v. 15, n.5, p. 567- 575, 2002.

REDDY, V.; URROJ, A.; KUMAR, A. Evaluation of antioxidant activity of some plant extracts and their application in biscuits. Food Chem., Amsterdam, v. 90, n.1-2, p. 317-321, 2005.

ROSS, R.G.; SELVASUBRAMANIAN, S.; JAYASUNDAR, S. Imunomodulatory avtivity of Punica granatum in rabbits - a preliminary study. $J$. Ethnopharmacol., Orlando, v.78, n.1, p. 85-87, 2001.

ROSSING, D.; KAHL, R.; HILDEBRANDT, A. G.Effect of synthetic antioxidants on hydrogen peroxide formation, oxyferro cytocrome $\mathrm{P} 450$ concentration and oxygen consumption in liver microssomes. Toxicology, Amsterdam, v. 34, n.1, p. 67-77, 1985.

ROBARDS, K.; PRENZLER, P.D.; TUCKER, G.; SWATSITANG, P.; GLOVER, W. Phenolic compounds and their role in processes in fruits. Food Chem., Amsterdam, v.66, n.4, p. 401-436, 1999.

SALLAM, K.H.; ISHIOROSHI, M.; SAMEJIMA, K.. Antioxidant and antimicrobial effects of garlic in chicken sausage. Lebensm.- Wiss. Technol., London, v. 37, n.8, p. 849-855, 2004.

SCHUBERT, S.Y.; LANSKI, E.P.; NEEMAN, I. Antioxidant and eicosanoid anzyme inhibition properties of pomegranate seed oil and fermented juice flavonoids. $J$. Ethnopharmacol., Orlando, v.66, n.1, p.11-17, 1999.

SINGLETON, V.L.; ORTHOFER, R.; LAMUELARAVENTÓS, R.M. Analysis of total phenols and other oxidation substrates and antioxidants by means of FolinCiocateau reagent. Methods Enzymol., San Diego, v. 299, p. $152-178,1999$.

TORRES, D.E.G.; MANCINI, D.A.P.; TORRES, R.P.; MANCINI-FILHO, J. Antioxidant activity of macambo (Theobroma bicolor L.) extracts. Eur. J. Lipid Sci. Technol., v. 104, p. 278- 281, 2002. 
VIDAL, A.; MOTIDOME, M.; MANCINI-FILHO, J.; FALLARERO, A.; MODORI, M.; BRANDÃO, L.M.; LAPA, A.J. Actividad antioxidante y ácidos fenólicos del alga marina Bryothamnion triquetrum (S.G. Gmelin) Howe. Rev. Bras. Cienc. Farm., São Paulo, v.37, n.3, p.373-382, 2001.

WÜRTZEN, G.; OLSEN, P. Chronic study on BHT in rats. Food Chem. Toxicol., Amsterdam, v. 24, n. 10-11, p. 1121-1125, 1986.
ZHOU, K.; YU, L.. Effects of extraction solvent on wheat bran antioxidant activity extimation. Lebensm-Wiss. Technol., London, v. 37, n.4, p. 717- 721, 2004.

Recebido para publicação em 28 de abril de 2006. Aceito para publicação em 03 de outubro de 2006. 